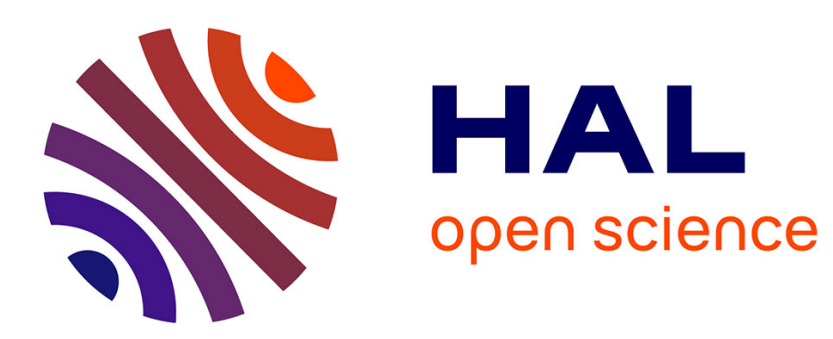

\title{
Violence symbolique et statut du politique chez Pierre Bourdieu
}

\author{
Lahouari Addi
}

\section{To cite this version:}

Lahouari Addi. Violence symbolique et statut du politique chez Pierre Bourdieu. Revue Française de Science Politique, 2001, 51 (6), pp.949-963. halshs-00398864

\section{HAL Id: halshs-00398864 https://shs.hal.science/halshs-00398864}

Submitted on 25 Jun 2009

HAL is a multi-disciplinary open access archive for the deposit and dissemination of scientific research documents, whether they are published or not. The documents may come from teaching and research institutions in France or abroad, or from public or private research centers.
L'archive ouverte pluridisciplinaire HAL, est destinée au dépôt et à la diffusion de documents scientifiques de niveau recherche, publiés ou non, émanant des établissements d'enseignement et de recherche français ou étrangers, des laboratoires publics ou privés. 
Violence symbolique et statut du politique dans l'œuvre de Pierre Bourdieu[1]

Lahouari ADDI[2]

Professeur à l'IEP de Lyon, Ceriep et Gremmo

In Revue française de science politique, décembre 2001

Résumé

Théorie globale, la sociologie de Pierre Bourdieu contient une dynamique politique que libère la conflictualité sociale généralisée appréhendée à travers les concepts de domination, de pouvoir, de violence symbolique, etc. Cependant la nature de ces concepts, en particulier celui de violence symbolique, donne au politique un statut ambigu en raison de leur incapacité à construire une théorie de l'État fondée sur l'exercice du monopole de la violence physique. Exercée par n 'importe quel agent, dans les espaces public et privé, la violence symbolique est caracté ristique d'une situation infra-étatique. Forgée à l'occasion de travaux d'anthropologie menée en Kabylie, elle contredit la théorie des champs, expression de la différenciation des pratiques sociales et de l'institutionnalisation des formes du politique dans les sociétés modernes. L'absence d'une frontière précise chez Bourdieu entre la sphère privée et la sphère publique a été un obstacle à penser l'État de droit et les libertés publiques que les luttes sociales ont conquises contre l'absolutisme du pouvoir.

Abstract: Symbolic violence and the status of politics in Pierre Bourdieu

A global theory, Pierre Bourdieu's sociology contains a political dynamic liberated by the generalized conflict understood via the concepts of domination, power, symbolic violence, etc. the nature of these concepts, in particular that of symbolic violence, gives however politics an ambiguous status because of their inadequacy for constructing a theory of the state founded on the exercise of the monopoly of physical violence. Exercised by everyone, in both the public and the private sphères, symbotic violence charactérizes an infrastate situation. Stemming front anthropological research undertaken in Kabylie (Àlgeria), it contradicts field theory, the expression of the differentiation of social practices and of the institutionalization of the forms of politics in modem societies. The absence of a précise boundary in Bourdieu 's work between the private and the public sphères has been an obstacle to conceiving the rule of law and the public liberties conquered through social struggles against the absolutism of power.

Sommaire

- Introduction

- I- Violence symbolique et coercition physique

- II- Typologie de la domination et nature de la violence

- III- Le staut ambigu du politique

- Conclusion

Texte intégral

\section{Introduction}


De par sa propension à penser le social dans sa globalité, la théorie de Pierre Bourdieu intègre l'objet du politique dans ses différents aspects à travers des concepts constitutifs du rapport politique tels que domination, pouvoir symbolique, légitimité, violence, etc. La mécanique conceptuelle du modèle souffre néanmoins d'ambiguïtés théoriques et méthodologiques qui empêchent de fonder une théorie de l'État comme puissance publique disposant d'un pouvoir fondamentalement différent de tous les autres pouvoirs. Réduit à un marché électoral, le champ politique n'est alors constitué que de " représentants » profitant de leurs positions dominantes et une masse d'agents hiérarchisés que les spécialistes des sondages - véritables ventriloques - font parler pour ajuster les demandes aux offres des entrepreneurs politiques. La comparaison avec le marché où se rencontrent l'offre des partis en concurrence et la demande des consommateurs inégalement armés pour choisir, pour illustrative qu'elle soit, ne permet pas de faire ressortir l'originalité du pouvoir politique, celle d'édicter des règles ayant force de loi et s'imposant à tous.

Les écrits de Bourdieu relevant de la sociologie politique traitent soit des sondages, de leurs constructions méthodologiques, de l'hétérogénéité des statuts des interviewés, de leur inégale maîtrise du discours, etc. pour montrer que l'opinion publique est une construction statistique -un artefact- et non une réalité sociologique ; soit de la représentation, dans une double perspective rousseauiste et néomachiavélienne, pour mettre en garde contre les limites de la démocratie électorale qui laisse une marge de manœuvre très large aux représentants, obéissant à une logique propre, celle de défendre d'abord leurs intérêts et de ne représenter qu'eux-mêmes[3]. Cette analyse n'épuise cependant pas l'objet du politique renvoyant au fondement de l'autorité et à la séparation des espaces public et privé. La raison de cette faiblesse est à relier à la structure du modèle construit autour de la violence symbolique impossible d'être monopolisée par quelque institution que ce soit. Dans cet article, nous montrerons la différence entre la violence symbolique et la coercition physique et ses consé quences sur l'apparition de l'État, l'échec de Bourdieu à construire une typologie de la violence en relation avec les modes traditionnel et moderne de domination, et enfin l'ambiguïté du politique - censé chez cet auteur être différencié - qui recouvre tout l'espace social, ce qui remet en cause la théorie des champs. La conflictualité généralisée du modèle donne au politique un caractère infra-étatique que la sociologie politique avait pourtant dépassé grâce aux problématiques de Machiavel, Hobbes, Rousseau, Marx, Weber...

\section{I- Violence symbolique et coercition physique}

Provenant de la réflexion sur les relations personnalisées de dépendance acceptées comme naturelles et légitimes par celui qui les subit, la notion de violence symbolique, constitutive $d u$ paradigme anthropologique kabyle[4], est supposée s'opposer à (ou se différencier de) la coercition physique, dans le sens où elle est une pression dont l'agent n'a pas conscience. Mécanisme fondamental de la reproduction de la domination dans l'approche de Bourdieu, elle est donc le présupposé de l'ordre social (inégalitaire) et de l'extorsion du surplus et de l'exploitation qui permet aux dominants de faire l'économie de la domination physique et brutale, dans la mesure où il y a complicité de tout le groupe ou mensonge du groupe à luimême. Cette idée de la participation des dominés à leur domination est récurrente chez Bourdieu pour qui «...le langage d'autorité ne gouverne jamais qu'avec la collaboration de ceux qu'il gouverne, c'est-à-dire grâce à l'assistance des mécanismes sociaux capables de produire cette complicité, fondée sur la méconnaissance qui est au principe de toute autorité 
»[5]. Constitutive du lien social dont elle cache la conflictualité, la violence symbolique assure la paix civile dans une structure inégalitaire des statuts, d'où l'importance cruciale du pouvoir symbolique qui, malgré son caractère arbitraire mais caché, obtient l'obéissance sans recourir à la force ni physique ni économique. Compte tenu de son importance, il est alors nécessaire de la définir de manière rigoureuse avant de lui faire jouer le rôle conceptuel qui est le sien : est-elle tout ce qui est différent de la violence physique ? Est-ce seulement celle utilisée dans la société traditionnelle sur la base des croyances des agents ?

Si le contenu théorique du concept est analysé et si sa validité est évaluée sur le terrain historique et dans les pratiques sociales relatives à ses manifestations, il serait facile de s'apercevoir que sa capacité analytique est limitée et que son usage généralisé dans le discours rappelle singulièrement celui de la notion d'idéologie dont la seule évocation par le marxisme dans les années 1960 donnait l'illusion de l'explication de la reproduction sociale et de ses contradictions. En effet, à y réfléchir, la violence symbolique se loge dans toute interaction sociale, et l'individu y est soumis dès le réveil du matin et soumet d'autres individus, le plus souvent sans en être conscient. Finalement, tout acte commis par un individu sans que le motif ne soit pur plaisir se déroule sous la pression de la violence symbolique : aller à l'école ou au travail, préparer le lit conjugal, servir un jus à son épouse, etc. Il est alors difficile de tracer une limite précise entre un acte commis volontairement et librement et un acte commis sous la contrainte de la violence symbolique, du fait même que celle-ci suppose le consentement de celui sur lequel elle s'exerce. Mais peut-on encore parler de violence dès lors qu 'il y a consentement ? La violence ne commence-t-elle pas seulement où l'adhésion n'est pas acquise ? La violence symbolique n'est-elle pas seulement le résultat du regard extérieur ? N'est-elle pas un effet d'optique du regard ethno-centriste [6] ? Une relation inégalitaire n'apparaît comme telle que pour celui qui ne la considère pas comme allant de soi. Autrement dit, pour la faire accepter par celui qui la subit et qui la considère comme naturelle, il n' y a pas besoin de recourir à la violence, symbolique ou non. Il suffit que l'individu croie aux valeurs dominantes, pour qu'il y adhère et pour qu'il participe à la reproduction de l'ordre social qui le domine sans qu'il ne soit conscient de cette domination. De ce fait, il n'est pas aisé de concevoir ce qu'est la violence symbolique et de la délimiter avec précision. Bourdieu est conscient de cette difficulté théorique mais il l'ignore, faisant comme si elle n'existait pas. Quelque peu scientiste, il considère que la violence symbolique repose fondalementalement sur la méconnaissance de son mécanisme par ceux qui la subissent. Tant que le mécanisme demeure caché, « le résultat du laissez-faire, complice du probable, apparaît comme un destin ; lorsqu'elle est connue, il apparaît comme une violence[7] ». Cela signifie-t-il que la violence symbolique est imposée par la force physique ? Ce qui serait absurde car si c'est le cas, qu'en est-il du consentement nécessaire à sa reproduction?

La difficulté provient de ce que la notion est insaisissable, à la différence de la violence physique qui se constate empiriquement, laissant des traces après avoir provoqué de la douleur physique. C'est pourquoi il a été possible de monopoliser celle-ci et non celle-là. En effet, le déroulement de l'histoire politique de l'Europe qui, depuis la Renaissance, a connu un mouvement d'absorption de la violence physique institutionnelle privée au profit d'un organe qui s'en est approprié le monopole de l'exercice, interdit la mise en parallèle des violences physique et symbolique, en raison de la nature même des différences entre les deux catégories. La différenciation sociale, comme mouvement historique, a accompagné la construction de l'État autour de la monopolisation progressive de l'exercice de la violence physique, à l'exclusion de la violence symbolique qu 'aucun individu et aucune institution ne peut monopoliser[8]. Il faut se rappeler que la construction de l'État - comme monopole-est en relation avec la contrainte sur le corps[9] et non sur les âmes. Si Luther est considéré comme 
un penseur moderne, c'est précisément parce qu'il a été parmi les premiers à distinguer la liberté extérieure de la liberté intérieure du chrétien, la première susceptible d'être limitée par la violence physique dont le monopole est reconnu au pouvoir politique, la seconde relevant de la conscience individuelle échappant à toute autorité constituée[10].

Dans la modernité, la coercition de l'État est physique, dans le respect de la liberté de conscience arrachée à l'Église qui a prétendu, par le passé, la limiter pour garantir le bonheur terrestre et céleste des croyants et même des incroyants. L'échec de l'Église à contrôler l'espace public - comme de toute autre institution religieuse - provient précisément de ce que la violence symbolique n'est pas susceptible d'être monopolisée. Exercée par des institutions ou des individus privés, il est difficile de la cerner juridiquement sans tomber dans la violation des libertés individuelles et publiques. II convient de prendre la mesure objective de la différence entre les deux types de violence, car tandis que la violence symbolique a besoin du consentement de celui qui la subit, la violence physique s'exerce toujours contre le gré d'une personne que nous supposons normalement constituée. Un individu peut porter plainte pour coups et blessures en apportant la preuve matérielle, mais il ne peut porter plainte pour violence symbolique dans la mesure où celle-ci n'est opératoire que s'il y a consentement et adhésion volontaire aux croyances qui la justifient. Les exemples ne manquent pas pour illustrer cette singularité qui rend difficile la législation sur les sectes, sur les écoles dites libres ou encore sur le port du foulard islamique. Ce que la législation peut faire, c'est de protéger des mineurs ou des handicapés, mais elle ne peut protéger des personnes contre leur propre volonté.

Le caractère insaisissable de la violence symbolique permet de mieux comprendre le rôle de l'État comme organe détenteur du monopole de l'exercice de la violence physique et uniquement celle-ci. Ce dernier est d'abord un appareil de coercition disposant d'instruments pour obliger les individus à respecter, par la contrainte des corps, allant de l'emprisonnement à l'exécution capitale, les règles qu'il édicté et qui ont force de loi. Il est aussi producteur de violence symbolique (école, droit...), mais il n'en a pas le monopole puisque des institutions privées l'exercent aussi (familles, sectes, différents pouvoirs sociaux, etc.) sans qu'il puisse l'interdire. Autorité souveraine détenant le monopole de la violence physique, l'État ne renonce pas pour autant à la violence symbolique, tout en sachant qu'il n'en aura jamais le monopole. Il essaie alors de remonter à la source, c'est-à-dire d'inculquer aux jeunes générations une instruction publique dans le cadre de l'éducation nationale pour se donner les chances de concurrencer, c'est-à-dire de délégitimer, tout autre pouvoir social ayant une influence sur la société[11]. Il tente en quelque sorte d'inculquer aux jeunes générations des habitus sans lesquels la violence symbolique serait insupportable, et donc inacceptable, et qui, surtout, feront l'économie de l'usage de la coercition physique. Ces habitus sont ce que l'on peut appeler la culture étatique, le respect - ou la crainte-de l'État sans lesquels l'autorité a peu de chance de se faire obéir.

Ce n'est ni une coïncidence ni un hasard si l'auteur de la problématique du monopole de l'exercice de la violence ( $M$. Weber) est simultanément celui de la théorie du « désenchantement du monde » qui, toutes deux, appartiennent au même mouvement historique de sécularisation par lequel les hommes prennent conscience de leur souveraineté - capacité de faire des lois - confiée à un organe dont la prérogative est d'assurer le maintien de l'ordre public par la police et les tribunaux. Car le « désenchantement du monde » est l'écroulement des croyances qui justifiaient la violence symbolique de la société traditionnelle, c'est le reflux du système mythico-rituel par lequel se reproduisaient la hiérarchisation et l'inégalité de la société traditionnelle, libérant un potentiel conflictuel menant à l'anarchie à laquelle met fin 
l'État, organe interdisant à quiconque de recourir à la force physique contre autrui. C'est toute la nuance entre les notions de légitimité et de légalité, la première étant du côté de la société civile productrice de valeurs donnant sens, et la seconde du côté de l'État se limitant à assurer la sécurité des citoyens. L'État ne dit pas ce qui est légitime - c'est à la société de le faire mais il dit ce qui est légal, traçant la frontière avec ce qui ne l'est pas sur le critère essentiel de l'utilisation de la violence physique et de l'atteinte à l'ordre public en relation avec la protection de la propriété privée. Car un État qui se pose comme source de légitimité est forcément un État autoritaire, voire totalitaire, porté à violer les consciences individuelles et les libertés publiques. C'est ce lien théorique et historique entre violence symbolique et coercition physique dans la genèse de la modernité politique qui manque dans l'œuvre de Bourdieu qui récuse du reste le modèle de l'État allant de Hobbes à Tilly, en passant par Marx, Weber et Elias, en pensant que la violence physique a été privilégiée à tort au détriment de la violence symbolique. Il écrit : "C'est la concentration du capital de force physique qui a été privilégiée dans la plupart des modèles de la genèse de l'État, des marxistes, enclins à considérer l'État comme un simple organe de coercition, à Max Weber et sa définition classique, ou de Norbert Elias à Charles Tilly [12] ». Bourdieu estime que l'État exerce le monopole de la violence symbolique [13] en soulignant que « les rapports de force les plus brutaux sont en même temps des rapports symboliques[14] ». Il paraît singulier que Bourdieu insiste sur la monopolisation par l'État de la violence symbolique alors que la réalité quotidienne donne de nombreux exemples de violence symbolique exercée par des institutions privées : entreprises, familles, associations, divers groupements, etc.

L'autre aspect appelant à discussion est l'évocation par Bourdieu de la genèse de l'État perçue comme « l'aboutissement d'un processus de concentration de différentes espèces de capital, le capital de force physique ou d'instruments de coercition (armée, police), capital économique, capital culturel ou, mieux, informationnel, capital symbolique, concentration qui, en tant que telle, constitue l'État détenteur d'une sorte de métacapital donnant pouvoir sur les autres espèces de capital et sur leurs détenteurs [15] ». Ce qui est gênant dans une telle approche est la nature a-historique des différents capitaux (force physique, culturel, économique...) qui préexisteraient à l'État et qui, en se concentrant, donneraient naissance à une autorité chargée d'établir un équilibre entre eux et de fixer le taux de change par lequel ils s'échangeront. Dans un autre passage, Bourdieu suggère que la genèse de l'État correspond à un processus de monopolisation de la force publique, alors que les historiens de l'État décrivent un processus inverse correspondant à une dé-privatisation du pouvoir qui, au fur et à mesure, s'affirme comme une puissance publique[16]. C'est en tout cas le modèle de la patrimonialisation forgé par Weber au sujet des monarchies de l'Europe médiévale, construit à l'aide de types-idéaux d'autorité correspondant à des phases historiques différentes (autorité charismatique versus autorité légale-rationnelle). La nature conceptuelle de la violence symbolique et physique ne permet de dresser, comme l'aurait voulu Bourdieu, une typologie de la domination ni dans une perspective théorique ni dans une perspective historique.

\section{II- Typologie de la domination et nature de la violence}

Bourdieu a tenté une typologie des violences en relation avec le mode de domination, cherchant à définir une société donnée par un type de violence. Classant les différentes sociétés sur un critère politique, Bourdieu explique qu'il existe deux types de domination : dans l'un, les relations sociales sont personnalisées, s'incarnant dans des figures humaines (la 
société traditionnelle), dans l'autre, elles sont désenchantées et institutionnalisées (la société moderne). " D'un côté, écrit-il, des relations sociales qui, n'ayant pas en elle mêmes le principe de leur reproduction, ne peuvent subsister qu'au prix d'une véritable création continuée ; de l'autre, un monde social qui, enfermant en lui-même le principe de sa propre subsistance, dispense les agents de ce travail incessant et indéfini d' instauration ou de restauration des relations sociales "[17]. À travers cette distinction, Bourdieu vise à construire une typologie de la domination, et donc de l'autorité, sur le critère de la nature de la violence : symbolique dans un cas, brutale dans l'autre. Mais il n'y arrive pas car dans le rapport d'autorité politique, la violence est symbolique en général et physique en particulier si nécessaire. Une règle juridique ou coutumière est respectée par une majorité sous l'effet d'une violence symbolique, mais si quelques individus ne s'y plient pas, ils s'exposent à une réaction brutale qui les y contraindra. Ainsi, il n'y aura pas de société fonctionnant seulement à l'une ou l'autre violence, et les règles sociales recourent dans tous les cas aux deux types de violence.

Il est vrai cependant que, dans la société traditionnelle, les relations sociales étant personnalisées (rapports de parenté, communautés locales restreintes...), la violence symbolique joue un plus grand rôle, d'autant que le monopole de l'exercice de la violence physique est quasi inexistant. Ceci signifie-t-il que la société moderne fait un usage moindre de la violence symbolique ? Certainement pas. Prenons, pour illustrer les deux cas de figure, l'exemple du khammessat qui a probablement suggéré à Bourdieu la notion de violence symbolique. Dans la société traditionnelle algérienne, deux personnages - le khammès et le paysan - illustrent le mécanisme de la domination, instituant des relations personnalisées, incarnées dans des visages familiers. Mode d'extorsion du surplus, le khammessat est vécu comme une association, voire une amitié, autour de l'entreprise de mise en valeur des terres et de partage des bénéfices $\left(1 / 5^{\mathrm{e}}\right.$ contre $4 / 5 \mathrm{e}$ au propriétaire). Fonctionnant avec le consentement des dominés (les khammès), ce mécanisme est régulé par une violence symbolique dont l'efficacité puise dans la croyance des agents dans sa légitimité car la relation de dépendance, comme partout ailleurs, a besoin d'être idéologiquement justifiée pour être acceptée. Ce consentement n'est cependant pas spécifique à la société traditionnelle ; on le retrouve dans la société capitaliste, en particulier dans la relation salariale qui ne s'établit que si le salarié a le sentiment de vendre librement sa force de travail au patron qui remploie contre un salaire que parfois il négocie, relation fonctionnant à la violence symbolique comme celle qui lie le khammès au paysan, et que l'on retrouve du reste dans toute hiérarchie sociale dans le passé ou le présent. II est intéressant de constater que le khammès percevait, tout au moins au début, le salariat comme une exploitation éhontée de l'homme par l'homme, tout en considérant le khammessat comme une relation d'hommes libres se faisant mutuellement confiance. Bourdieu cite le cas d'ouvriers algériens dépaysannés qui se plaignaient de ce que le colon à la ferme ou le patron à l'usine les exploite, en utilisant une expression suggestive ; yehrat alia (il me laboure dessus ou il laboure sur moi) [18]. Les anciens khammès vivaient le salariat comme une oppression, alors qu'ils se sentaient plus libres dans le khammessat[19]. Mais ce qu'il y a de plus intéressant à constater, c'est que, après la généralisation de l'échange marchand, le khammessat apparaît comme une forme d'esclavage et le salariat comme un rapport d'hommes libres. Dans l'approche de Bourdieu, pour qu'il y ait passage d'un système à un autre, il faut que l'habitus du salariat se substitue à celui du khammessat pour que disparaisse le sentiment d'étrangeté du monde social.

La tentative de typologie des violences provient probablement de la perception différente du khammessat et du salariat par les intéressés, sur la base de leur habitus, d'où la conclusion hâtive que le premier rapport social serait régulé par la violence symbolique, tandis que le 
second le serait par la violence physique. Bourdieu a été en effet tenté d'affirmer que le mode privilégié de la reproduction de la société traditionnelle est celui de la violence douce et du consentement des dominés à l'ordre social inégalitaire. Il n'est cependant pas sûr que cette hypothèse soit confirmée par les travaux d'histoire portant sur des périodes pré-étatiques où la violence physique privée était le moyen le plus commun de se protéger des agressions et de défendre ses biens, son honneur et sa famille. Mais, pressentant cette remarque, il consent que la société traditionnelle a recours aux deux formes de violence, écrivant : " Il ne faut donc pas voir une contradiction dans le fait que la violence est à la fois plus présente et plus masquée. C'est parce qu'elle ne dispose pas de la violence implacable et cachée des mécanismes objectifs qui autorisent les dominants à se contenter de stratégies, souvent purement négatives, de reproduction, que cette économie a recours simultanément à des formes de domination qui, du point de vue de l'observateur contemporain, peuvent paraître tout à la fois plus brutales, plus primitives, plus barbares ou plus douces, plus humaines, plus respectueuses de la personne »[20]. Autrement dit, les «mécanismes objectifs » de la société moderne droit, école, marché... - sont-ils constitutifs de violence symbolique ou non ? Si l'on considère l'ensemble des travaux de Bourdieu, la réponse ne fait aucun doute : la société moderne fonctionne à la violence symbolique, en faisant très peu recours à la violence physique utilisée contre une minorité dans le cas de violations de l'ordre public.

Au-delà de la circularité du raisonnement qui explique la reproduction de la société traditionnelle par un manque par rapport à la société moderne, Bourdieu postule implicitement que la violence dans la société moderne est plus physique que symbolique, en tout cas « implacable et cachée ». Ce qui pose alors le problème du statut de la violence - symbolique ou physique - dans la société moderne et, au-delà, le statut du politique, laissant apparaître une contradiction fondamentale dans les écrits de Bourdieu relatifs la société française analysée à travers la violence symbolique générée par ses institutions. On ne peut, en effet, soutenir, d'une part, que la violence symbolique dépérit au fur et à mesure de la modernisation[21] et, de l'autre, qu'elle est hégémonique, diffusée par l'école, le marché, l'État, etc. De deux choses l'une : ou bien la société française est moderne et recourt marginalement à la violence symbolique dans le schéma proposé par Bourdieu, ou bien, elle n'est pas moderne, et est régulée à un niveau infra-étatique par une violence symbolique généralisée.

Aucun des deux termes de l'alternative ainsi formulée ne correspond à la réalité, et c'est l'alternative elle-même qui n'est pas pertinente car la violence symbolique n'est spécifique ni à la société traditionnelle ni à la société moderne, et aucune domination, quelle que soit la période historique, ne peut se reproduire sans violence symbolique. En relation avec la légitimité qui l'institue et qui lui donne sa pertinence, elle est présente dans tous les types de domination car aucun ordre social n'a la capacité de se reproduire avec la seule violence physique caractéristique de l'état de guerre hobbésien. Constater que la société traditionnelle se reproduit par le biais de la violence symbolique décrite comme un mécanisme idéologique auquel adhèrent tous les agents - dominants et dominés - est une façon de souligner l' indifférenciation sociale et la non-autonomie des différents champs (religieux, politique, économique... ), à travers, notamment, le caractère généralisé de l'usage privé de la violence physique là où la violence symbolique ne suffit pas à assurer l'équilibre des intérêts conflictuels. Par exemple, en cas de meurtre, le village kabyle encourage la vengeance privée s'instituant comme mécanisme dissuasif. L'échec de la violence symbolique à garantir la sécurité trouve son prolongement dans l'usage privé de la violence physique contribuant à renforcer la violence symbolique plutôt qu'à s'y substituer. L'illusion que la société traditionnelle fonctionne à la violence symbolique, à l'inverse de la société moderne, provient de ce que la première, à la différence de la seconde, ne dispose pas d'un organe prétendant au 
monopole de l'exercice de la violence physique qui suppose l'autonomie d'un champ politique structuré autour de l'État doté d'appareils répressifs.

C'est en observant la Kabylie, société indifférenciée, que Bourdieu a forgé ses principaux outils conceptuels qu'il utilise pour analyser des sociétés modernes différenciées, d'où des problèmes d'ordre méthodologique. L'utilisation de la notion de violence symbolique dans des travaux consacrés à la France a été faite comme s'il n'y avait pas un organe exerçant le monopole de la violence physique ou comme si cet organe -l'État- exerçait un pouvoir social banal parmi tant d'autres, ou encore comme si la violence symbolique était du seul fait de cet État supposé en détenir le monopole. En somme, Bourdieu soumet la société moderne à la logique de la société traditionnelle, remettant en cause le postulat de la différenciation à laquelle il paraît adhérer à travers la théorie des champs. Certes, il est question de l'autonomie du politique, mais celui-ci n'est pas délimité, coïncidant avec tout l'espace social.

Prenant sa source dans le capital social, véritable pouvoir conférant à son détenteur une autorité légitime, la violence symbolique est le moyen par lequel la domination se reproduit dans une relation où l'investissement est tout autant social qu'économique. Le notable qui accumule du capital social doit dépenser du capital économique à cette fin puisque la règle est que le prestige se paie. " C'est ce qui fait, écrit Bourdieu, que, par un paradoxe qui est constitutif de ce mode de domination, le moyen mange la fin et que les actions nécessaires pour assurer la durée du pouvoir contribuent à sa fragilité » [22] Mais " le moyen mange la fin » est l'expression de la rationalité écono mique propre à la société capitaliste ; ce qui indique que Bourdieu n'étudie pas un type de domination en lui-même, mais le rapporte à un autre type posé tantôt explicitement, tantôt implicitement, comme une norme comparative, II fait référence à la rationalité du marché quand il analyse les pratiques sociales du village kabyle, mais il fait aussi référence au paradigme anthropologique kabyle quand il étudie la société française du dernier tiers du $20^{\mathrm{e}}$ siècle, à travers les catégories de capital social et de violence symbolique. Dans une comparaison incessante et croisée avec la société de marché, le paradigme anthropologique kabyle permet de saisir a contrario la dynamique de différenciation sociale que la modernité a enclenchée, et que de nombreux auteurs, parmi lesquels Marx, Tönnies, Durkheim, Weber..., ont analysée en utilisant des concepts différents. L'originalité de Bourdieu, par rapport à ces auteurs, est que le critère de distinction est essentiellement politique et est relatif à la forme de domination par laquelle se reproduisent les présupposés économiques et idéologiques de l'inégalité et de la hiérarchisation sociale.

Cette remise en cause de la différenciation prend en fait sa source dans l'identité d'approche de la société traditionnelle et de la société moderne, d'où l'universalité du politique ou, ce qui revient au même, la non-autonomie du champ politique induite par le concept central de la problématique - la violence symbolique - dont l'usage pose des problèmes relatifs à la cohérence interne de l'approche. La violence symbolique est au système social ce que la légitimité est à l'ordre politique que Bourdieu dissout dans l'espace social analysé comme champ de luttes généralisées et de compétitions permanentes. Sous l'effet du paradigme anthropologique kabyle, la problématique de Bourdieu appliquée à la France opère deux glissements : le premier, de la différencia tion sociale vers l'indifférenciation, contredisant la théorie des champs exposée par ailleurs ; le second, conséquence du premier, du champ politique vers l'espace social.

Ces deux glissements interdisent d'appréhender le politique dans son autonomie et surtout dans ses incarnations modernes, en premier lieu l'État. Au-delà de l'État, la question du politique se pose dans la théorie de Bourdieu qui refuse de le penser méthodologiquement en 
dehors de ses déterminations sociales, d'où ses critiques contre la « science politique » accusée de limiter, entre autres, le champ politique au champ du pouvoir, lui reprochant d'ignorer que les mécanismes qui légitiment celui-ci plongent leurs racines dans tout l'espace social « L'existence de mécanismes capables d'assurer la reproduction de l'ordre politique en dehors de toute intervention expresse autorise à ne reconnaître comme politique que celles des pratiques orientées vers l'acquisition ou la conservation du pouvoir qui excluent tacitement de la compétition légitime pour le pouvoir la maîtrise des mécanismes de reproduction. C'est ainsi qu'en se donnant pour objectif principal - comme aujourd'hui ce que l'on appelle la "science politique" - la sphère de la politique légitime, la science sociale a longtemps repris à son compte l'objet pré-construit que lui imposait la réalité »[23], Au-delà de la critique, somme toute justifiée de ce qu'on appelle le discours « Sciences po » (participant à l'illusion égalitaire de la démocratie électorale), et compte tenu du fait que la science politique ne se réduit pas à ce discours, il se pose le problème du statut du poli tique chez Bourdieu dont l'approche sociologique ne permet de penser ni l'État ni le droit dans leurs singularités historiques et méthodologiques.

\section{III- Le statut ambigu du politique}

Il y a schématiquement, en sociologie, deux grandes traditions : l'une, privilégie la cohésion sociale et les facteurs d'intégration de la société, l'autre met l'accent plutôt sur la conflictualité et les facteurs de désintégration. En général, la théorie politique, liée au deuxième courant, est inspirée par le pessimisme anthropologique qui présuppose que les rapports sociaux portent en eux les germes de la domination et de la violence et que l'Etat est nécessaire face aux risques d'éclatement de la collectivité et aux menaces potentielles d'anarchie[24]. Dans cette classification, Bourdieu occupe une position atypique car, bien qu'appartenant à la tradition de la conflictualité, le politique chez lui, intimement lié au social, n'est pas un objet autonome. Dans la mesure où toute la société se constitue comme le théâtre d'une violence symbolique généralisée pour l'acquisition et le contrôle des biens rares, le politique se caractérise par une situation structurellement infra-étatique de par l'absence du monopole de l'exercice de la violence symbolique dont nous avons souligné supra la spécificité. La société étant un champ de bataille ou la violence symbolique est manifeste dans le moindre recoin du lien social, il est difficile alors de situer et d'autonomiser le politique, et donc de l'institutionnaliser. Si l'on définit le politique comme étant l'espace des conflits ayant pour enjeu la préservation et la conquête de l'exercice de l'autorité publique, il n'y a alors pas de politique dans la sociologie de Bourdieu où la conflictualité est généralisée et d'essence privée. Cette caractéristique n'est pas une insuffisance théorique de son approche mais découle de la méthodologie et des hypothèses qui construisent une problématique dans laquelle la lutte pour le pouvoir est générale comme dans l'état de nature de Hobbes sans qu'il n'y ait aucune perspective pour que le pouvoir privé évolue vers une forme d'autorité publique [25] La société y est une articulation d'intérêts conflictuels qui fonctionne à la violence symbolique reproduisant la domination sans possibilité d'y mettre fin d'une manière ou d'une autre. Ce pessimisme politique-qui ne permet la fin de la violence ni par l'état civil à la Hobbes ni par l'État du peuple à la Marx-envahit toute l'œuvre de Bourdieu dans laquelle il n'y a pas de place pour un projet politique cohérent, et se distingue du pessimisme anthro pologique sur lequel la philosophie politique de Hobbes à Hegel, en passant par Spinoza, Rousseau et Kant, a construit la théorie de l'État

Ayant choisi de donner à la violence symbolique le rôle de régulateur principal de la hiérarchie sociale et des rapports de domination qui la dessinent, Bourdieu condamne le 
politique à la sphère privée, c'est-à-dire à un niveau infra-étatique. Mettant en avant la violence symbolique généralisée de la part d'individus privés, il évoque rarement le monopole de l'exercice de la violence physique, du fait de la prégnance, dans son approche, du paradigme anthropologique kabyle. La violence symbolique envahit tout l'espace social, et sa confusion avec la violence physique l'empêche d'élaborer une théorie politique ou d'analyser la genèse de l'État à travers le processus historique d'absorption de la violence privée institutionnelle, dont la finalité a été d'assurer la sécurité à ceux qui lui prêtent allégeance, ce qui signifie l'interdiction du recours à la force physique pour résoudre des conflits privés. Survalorisant la conflictualité symbolique disséminée dans tout l'espace social, Bourdieu se prive d'une théorie de l'État parce que aucun organe ne peut mettre fin à la violence symbolique, en raison même de son caractère non monopolisable. Que Bourdieu souligne que l'espace social est celui de la violence symbolique, il a raison ; mais il faut être conscient que cette posture n'est porteuse d'aucun dépassement, d'aucun avenir qui y met trait fin ou qui la réglementerait. L'État exerçant le monopole de la violence symbolique n'existera pas, fort heureusement [26] Il n'est pas possible d'extrapoler à partir de la violence physique et de construire une théorie politique sur la base de la violence symbolique. Aucun État ne peut promettre la protection contre la violence symbolique ou de mettre fin à son usage forcément privé. L'impossibilité du monopole de l'exer cice de la violence symbolique par quelque institution que ce soit, rend inapte l'approche de Bourdieu à produire une théorie de l'État ou à analyser celui existant dans les sociétés modernes, qu'il réduit du reste à un pouvoir social parmi d'autres, se frayant un chemin dans la jungle des violences symboliques concurrentielles pour satisfaire les intérêts de son personnel.

Condamné à la sphère privée-qui devient hégémonique au point où il n'y a pas de place pour la sphère publique - le politique est exprimé par des rapports de domi nation d'individus, à l'instar de l'espace domestique, sans jamais évoluer vers des institutions publiques qui les réguleraient. Alors de deux choses l'une : ou bien cette approche relève de la sociologie politique dont elle serait une branche prenant en charge les pratiques conflictuelles non étatiques ; ou bien il n'y a pas de place pour une sociologie politique ayant pour objet la spécificité du politique. Les travaux de Bourdieu suggèrent que la sociologie politique n'a pas de raison d'être car la sociologie est d'emblée politique, illustrant cette hypothèse par l'exemple de la sociologie de la famille qui montre le caractère conflictuel des relations entre époux et la compétition autour du contrôle des ressources de l'espace domestique. «Peut-être, écrit-il, parce que la question des fondements économiques du pouvoir domestique y est abordée avec plus de réalisme qu'ailleurs... peut-être parce que, du même coup, les représentations et les stratégies y sont plus proches de la vérité objective, la société béarnaise suggère que la sociologie de la famille, si souvent livrée aux bons sentiments, pourrait n'être qu'un cas particulier de la sociologie politique : la position des conjoints dans les rapports de force domestiques et leurs chances de succès dans la concurrence pour l'autorité familiale, c'est-à-dire pour le monopole de l'exercice légitime du pouvoir dans les affaires domestiques, ne sont jamais indépendants du capital matériel et symbolique (dont la nature varie selon les époques et les sociétés) qu'ils détiennent ou qu'ils ont apporté »[27]. La question est alors de savoir quel est, selon Bourdieu, l'objet originel de la sociologie politique ? Si cet objet est constitué par toute relation d'autorité, par tout pouvoir social, il est alors impossible de différencier la sociologie politique de la sociologie en général, et de distinguer celle-ci de l'anthro-pologie politique, en raison précisément du refus de spécifier le pouvoir politique par rapport à tous les pouvoirs sociaux que génère la vie en société.

Prêter à tout pouvoir social les caractères du pouvoir politique, c'est refuser de voir la singularité de ce dernier qui oblige à l'obéissance par le recours à la force si nécessaire - ce 
qui n'est pas le cas des autres pouvoirs sociaux, à l'exception de la relation de l'esclavage -, qui impose l'allégeance en s'arrogeant la prérogative de désigner l'adversaire, et enfin qui trace la frontière entre les espaces privé et public soumis à des règles juridiques dont il assure le respect. Le patron a une autorité sur l'ouvrier, le père sur ses enfants, le curé sur ses ouailles, mais aucune de ces autorités ne ressemble à celle du maire ou du préfet sur les administrés à qui il est imposé par la force, justifiée par le droit, des règles ayant force de loi. Ce que Bourdieu n'a pas vu, c'est que de tous les pouvoirs, seul le pouvoir politique est souverain, et c'est cela qui fonde la différence et la singularité du politique[28]. Le pouvoir politique est d'essence publique - à la différence des pouvoirs sociaux qui sont privés-même s'il arrive que des fonctionnaires, qui l'exercent par délégation, le détournent dans certains cas pour des usages privés, ce qui est formellement condamné par la loi. En niant la spécificité du pouvoir politique, Bourdieu efface la frontière entre le public et le privé, constitutive du politique et sans laquelle la notion d'État, garant de l'ordre public, serait inintelligible[29]. Qu'un représentant use et abuse de ses prérogatives pour servir ses intérêts ne nous rensigne pas d'où lui vient l'autorité que lui reconnaissent les administrés et n'aide pas à distinguer la ligne de démarcation entre ses actions publiques et privées. Bourdieu n'évoque jamais ou presque la paix civile nécessaire à la reproduction de la société, les libertés publiques et individuelles, le droit, la souveraineté du corps électoral, l'espace public, etc. Si ces thèmes ne sont pas abordés, ce n'est pas parce qu'il les a négligés ; c'est principalement parce que son approche méthodologique ne permet pas de penser le politique comme construction institutionnelle visant à assurer un ordre social interdisant l'usage privé de la violence physique dans une société formée d'intérêts antagoniques susceptible de basculer dans la guerre de tous contre tous. En fait, ce qui pose problème, c'est le statut du concept de « violence symbolique » sur lequel il est difficile de bâtir une théorie politique et même de penser le politique dans la mesure où il n'est opératoire qu'à condition qu'il soit rattaché à celui de légitimité auquel il s'articule. Présente dans les espaces privé et public, la violence symbolique a cette particularité de nier la frontière entre les deux espaces et de mettre l'État en compétition avec les autres acteurs, comme s'il n'avait pas le monopole de l'exercice de la violence physique. En insistant sur l'exercice de la violence symbolique par l'État, Bourdieu oublie que celui-ci revendique avec plus ou moins de succès le monopole de la violence physique qu'il refuse à tout autre pou voir, en ayant sa police, sa prison, son droit, son armée, etc. F. Bon et Y. Schemeil $\stackrel{[30]}{\longrightarrow}$ attirent l'attention sur la référence excessive à la violence symbolique de l'État au détriment de la coercition physique passée sous silence parce que... taken for granted.

\section{Conclusion}

En conclusion, enfermé dans le paradigme anthropologique kabyle à l'intérieur duquel le politique n'est ni spécifié ni autonome des relations d'autorité qui le véhiculent et qui l'expriment, Bourdieu refuse de distinguer le pouvoir politique des autres pouvoirs. C'est ce qui introduit une contradiction méthodologique dans la théorie des champs, et ce qui explique aussi qu'il n'y a chez lui ni une théorie du droit ni une théorie de l'État, siège de l'autorité publique censée incarner l'unité de la collectivité politique et dont la prérogative essentielle est de faire la loi et de l'appliquer en vue de la paix civile dans une société aux intérêts matériels et idéologiques conflictuels. La conséquence est qu'il n'y a pas chez lui de séparation entre sphère publique et sphère privée ; il y a un seul espace, celui des luttes privées puisant dans différents registres leurs ressources de légitimation. La contradiction public-privé constitutive du politique est absente, d'où l'inexistence d'une théorie de l'État qu'expliquent en partie l'indifférenciation sociale dans laquelle il fait retomber la société 
moderne, et la pra tique généralisée de la violence symbolique dans l'espace social, rappelant mutadis mutandis, l'état de nature.

II est intéressant à ce niveau de comparer Bourdieu à Durkheim dans une perspec tive de sociologie politique. Si le projet sociologique de celui-ci était de montrer la voie à une meilleure intégration de la société, celui du premier est d'indiquer comment l'agent est en conflit perpétuel avec son environnement social. II n'y a pas une problé matique du politique chez Durkheim parce que sa sociologie, s'enracinant dans la conscience collective incarnant la société conçue comme substance, exclut le conflit du lien social pensé comme générateur d'anomie. À. l'inverse, il n'y en a pas chez Bourdieu parce que, voulant éviter les pièges du holisme, il élimine les institutions et survalorise l'agent à travers une conflictualité enfermée dans le champ clos d'intérêts privés. Durkheim évite le politique en privilégiant l'unité de la société considérée comme réalité empirique sui generis ; Bourdieu limite le politique à une conflictualité individuelle généralisée et non institutionnalisée. L'un analyse la société par le haut, comme conscience collective homogène n'ayant besoin de l'État que pour des tâches administratives ; l'autre par le bas comme pratique d'agents la reproduisant dans l'anarchie conflictuelle par la violence symbolique dont seul le rapport de forces des interactions individuelles fixe les limites .

Notes

[1] Cet article est une version remaniée d'un chapitre d'un ouvrage à paraitre: Sociologie et anthropologie chez Pierre Bourdieu : le paradigme anthropologique kabyle et ses consé quences théoriques

[2] Lahouari Addi est professeur de sociologie politique à l'Institut d'études politiques de Lyon. Il est également chercheur au CERIEP (Centre de recherche de l'Institut d'études politiques) et au GREMMO (Groupe de recherche et d'étude sur la Méditer ranée et le Moyen-Orient). Collaborateur au Monde diplomatique, il est spécialiste du système politique algérien et a publié L'Algérie et la démocratie, Paris, La Découverte, 1995 ; Les mutations de la société algérienne, Paris, La Découverte, 1999. Il a par ailleurs contribué à divers ouvrages, notamment " Political Islam and Democracy : The Case of Algeria », dans Axel Hadenius, Democracy's Victory and Crisis, Cambridge, Cambridge University Press, 1997 ; «Algeria and me Dual Image of the Intellectual », dans Jeremy Jennings, Anthony Kemp-Welch, Intellectuals in Politics, Londres, Rout-ledge, 1997 ; " Algeria's Agony, Algeria's Army », ForeignAffairs, août 1998. Il vient. d'organiser un colloque international sur l'Anthropologie du Maghreb (Institut d'études politiques de Lyon, 20 et 21 septembre 2001). Il achève actuellement un ouvrage issu d'un séminaire de DEA intitulé Anthropologie et sociologie chez Pierre Bourdieu. Le paradigme anthropologique kabyle et ses conséquences théoriques, Paris, La Découverte, à paraître en 2002 (CERIEP, Institut d'études politiques de Lyon, 14 Avenue Ber-thelot,69007 Lyon <Lahouari.Addi@univ.-Lyon2.fr>)

[3] Encore que ces travaux sont d'inégale valeur dans la mesure ou, si ceux portant sur les sondages et l'opinion publique (" L'opinion publique n'existe pas». Les Temps modernes, 1970, et «Questions de politique >, Actes de ta recherche en science sociales., 16, sep tembre 1977) sont originaux, ceux, en revanche, portant sur la représentation politique (" La représentation politique. Éléments pour une théorie du champ politique». Actes de la 
recherche en sciences sociales, 38, février-mars 1981 ; « La délégation et le fétichisme politique ", ibid., 52-53, juin 1984, et enfin, dans le même numéro, " Espace social et genèse des "classes" ») ne font que répéter les néomachiavéliens et la critique de J.-J. Rousseau à l'endroit de la démocratie representative.

[4] Les principaux concepts de la sociologie de Bourdieu ont été forgés à la faveur de travaux d'anthropologie menés en Kabylie entre 1957 et 1962. Ce qui est ici appelé paradigme anthropologique kabyle est le modèle de village où les relations sociales, personnalisées, sont régulées par un sens de l'honneur, véritable capital, qu'il faut préserver et accroître par l'usage. forcément privé de la violence - symbolique et physique - qui reproduit une domination perçue comme légitime. Le sens de l'honneur libère une dynamique de distinction sociale qui donne un pouvoir à ceux qui se hissent au sommet de la hiérarchie sociale.

[5] P. Bourdieu, Ce que parler veut dire, Paris, Minuit, 1986, p. 113.

[6] C'est la question qu'avait su poser Montesquieu : «Comment peut-on être Persan ? » Cette question peut aussi être ramenée au niveau quotidien des relations interpersonnelles. Que de fois n'a-t-on pas entendu autour de nous une femme parler de son amie : "Comment fait elle pour le supporter? Moi je serais déjà partie »

[7] P. Bourdieu. "La mort saisit le vif », Actes de la recherche en sciences sociales, 32-33, 1980, p. 14

[8] La religion a prétendu au monopole de l'exercice de la violence symbolique, mais cela n'a été relativement efficace que dans le cadre de microsociétés locales s'identifiant à la reli gion (schéma de Durkheim dans Les formes élémentaires de la vie religieuse) et, dans ce cas, le monopole serait exercé au nom de la religion par tous les croyants, ce qui annulerait l'effet de monopole. Les sociétés musulmanes, aux prises avec l'islamisme, vivent présentement cette contradiction de l'exercice concurrentiel et généralisé de la violence symbolique - se prolon geant par des actes de violence physique, notamment contre les femmes - par tout un chacun s'investissant de l'autorité d'avoir la meilleure interprétation du Coran. Cette situation est aggravée par le fait qu'aucune institution en islam ne peut prétendre au monopole de dire ce qui est religieusement légitime, ce qui à coté d'autres facteurs plus politiques, perpétue le caractère infra-étatique des rapports sociaux

[9] Cf. les travaux de Michel Foucault, en particulier Surveiller et punir, Paris, Gallimard, 1978

[10] «Si donc ton Prince ou ton seigneur temporel, écrit Luther s'adressant au chrétien, t'ordonnait d'être pour le pape, de croire de telle ou telle façon, ou s'il t'imposait de te débarrasser de certains livres, tu diras ainsi : Lucifer n'a pas le droit de siéger à côté de Dieu. Mon seigneur, je vous dois obéissance quant à mon corps et mes biens. Donnez-moi des ordres dans les limites de votre pouvoir sur terre et je vous obéirai. Mais si vous m'imposez de croire et de rejeter les livres, je ne vous obéirai pas, car vous agissez là en tyran, vous outrepassez vos droits et donnez des ordres dans une sphère à laquelle ne s'étend ni votre juridiction ni votre pouvoir, etc. », M. Luther, Oeuvres, Genève, Labor \& Fides, vol. IV, 1958, p. 36.

[11] C'était le projet de Durkheim de renforcer la cohésion sociale par l'éducation publique chargée d'inculquer aux jeunes générations la laïcité qui leur permettra de s'autonomiser de la 
morale religieuse à laquelle devait être substituée une morale sécularisée. Il faut se rappeler que pour Durkheim la sociologie sera la science positive des mœurs, destinée à combattre l'anomie et à renforcer la cohésion sociale. Sociologie et socialisation vont de pair chez le fondateur de la sociologie française. Cf. É.Durkheim, L'éducation morale, Paris, F. Alcan, 1925, et aussi L'évolution pédagogique en France, Pans, F. Alcan, 1938.

[12] P. Bourdieu, Raisons pratiques, Paris, Le Seuil, 1994, p. 109

[13] « Anticipant sur les résultats de l'analyse, je dirai, dans une forme transformée de la formule célèbre de Weber,... l'État est un X (à déterminer) qui revendique avec succès le monopole de la violence physique et symbolique sur un territoire déterminé et sur l'ensemble de la population correspondante », ibid., p. 107

[14] ibid.,p. 124

[15] Ibid,p.109.

[16] « Je pourrais dire, en simplifiant beaucoup, que la construction de l'État dynastique, puis de l'État bureaucratique a pris la forme d'un processus de concentration de différentes espèces de pouvoir, ou de capital, aboutissant, dans un premier temps, à la monopolisation privée - par le soi - d'une puissance publique, à la fois extérieure et supérieure à toutes les puissances privées (les seigneurs, les bourgeois citadins, etc.) » : P. Bourdieu, Loïc J. D. Wacquant, Réponses, Paris, Le Seuil, 1992, p. 89. Le lecteur pourrait comprendre, à travers cette citation, que la dynamique historique du politique en Occident repose sur la privatisation d'une « puissance publique » qui aurait pré-existé à l'Etat, alors que c'est précisément l'inverse qui s'est produit si l'on se réfère au patrimonialisme qui identifiait le pouvoir comme une propriété privée et qui a, dans le temps, évolué vers des formes d'autorité publique

[17] P. Bourdieu, « Les modes de domination », Actes de la recherche en sciences sociales, 23, juin 1976, p. 126.

[18] Une autre expression dévalorisant le travail salarié est utilisée en Oranie : jarnat (de journalier, de quelqu'un qui n'a pas la garantie du lendemain)

[19] Cf. P. Bourdieu, Sayad Âbdelmalek, Le déracinement, Paris, Minuit, 1964

[20] Ibid, p. 128

[21] «S'il est vrai que la violence symbolique est la forme douce et larvée que prend la violence lorsque la violence ouverte est impossible, on comprend que les formes symboliques de la domination aient progressivement dépéri à mesure que se constituaient les mécanismes objectifs qui, en rendant inutile le travail d'euphémisation, tendaient à produire les dispositions désenchantées qu'exigeait leur développement ».ibid., p. 131

[22] Ibid.p.122

[23] P. Bourdieu. « Espace social et genèse des "classes" », art cité, p. 126

[24] Le courant de l'intégration posant l'unité de la société comme hypothèse de départ, le politique devient alors accessoire et non nécessaire 
[25] Nous avons vu dans la note 2, page 954, que Bourdieu fait dérouler l'histoire de l'État en partant d'une autorité publique préexistant à celui-ci vers sa privatisation

[26] Il est symptomatique que Bourdieu n'utilise que rarement l'expression " monopole de la violence symbolique ». Je ne l'ai trouvée que deux fois sous sa plume dans Réponses, op. cit., p. 87, dans "Espace social et genèse des "classes" », art cité, p. 7, et maintes fois uti lisée dans Raisons pratiques, op. cit

[27] P. Bourdieu, Le sens pratique, Paris, Misait, 1980, p. 264

[28] C'est pour avoir ignoré cette singularité du pouvoir politique, réduit à être le reflet mécanique du pouvoir social, que le marxisme vulgaire a dérivé vers le goulag, l' arbitraire et l'autoritarisme le plus brutal. Car il n'est pas vrai que tous les pouvoirs se valent et qu'ils soient identiques, pour l'unique raison que, si les pouvoirs sociaux sont privés, exercés par des individus défendant leurs intérêts propres, le pouvoir politique est d'essence publique, reposant sur la force du public qui met celle-ci à sa disposition s'il est considéré comme légitime

[29] Que cet ordre public soit à l'avantage des classes dominantes ne remet pas en cause cette définition de l'État qui a besoin, pour asseoir sa légitimité, d'apparaître comme neutre par rapport aux intérêts antagoniques de la société et surtout apparaître comme préoccupé essentiellement par le bien commun (common good) profitant à tous

[30] R Bon et Y. Schemeil attirent l'attention sur cette négligence en écrivant : " À force de vouloir mettre la violence symbolique dans toutes les pratiques sociales, écrivent-ils, Pierre Bourdieu en vient à oublier le rôle de la violence tout court, des institutions qui régulent son utilisation et donc à éliminer la spécificité du politique comme organisation de l'exercice de la violence légitime... Ainsi, la sociologie politique de Bourdieu ignore le maintien de l'ordre et les manifestations de rue, le lock-out et les grèves, la police, l'armée, la justice et les prisons ", $\mathrm{R}$ Bon, Y. Schemeil, « La rationalité de l'inconduite. Comprendre le statut du politique chez Revue française de science politique, 30 (6), 1980, p. 1203 